\title{
marges Marges
}

revue d'art contemporain Revue d'art contemporain

$21 \mid 2015$

Manifestes

\section{Le manifeste à l'écran entre la Nouvelle Vague et Dogme 95}

The manifesto on the screen between The New Wave and Dogma 95

\section{Carlos Tello}

\section{(2) OpenEdition}

\section{Journals}

Édition électronique

URL : http://journals.openedition.org/marges/1021

DOI : $10.4000 /$ marges. 1021

ISSN : 2416-8742

\section{Éditeur}

Presses universitaires de Vincennes

\section{Édition imprimée}

Date de publication : 1 octobre 2015

Pagination : 22-34

ISBN : 978-2_84292-441-6

ISSN : 1767-7114

\section{Référence électronique}

Carlos Tello, «Le manifeste à l'écran entre la Nouvelle Vague et Dogme 95 », Marges [En ligne], 21 | 2015, mis en ligne le 01 octobre 2017, consulté le 19 avril 2019. URL : http:// journals.openedition.org/marges/1021; DOI : 10.4000/marges.1021 


\section{Le manifeste à l'écran entre la Nouvelle Vague et Dogme 95}

L'apparition d'un manifeste au sein du cinéma constitue, pour les réalisateurs mais aussi pour les producteurs, critiques, spectateurs, etc., l'occasion - ou l'obligation - de prendre position. Parmi toutes les nuances possibles, ce qu'il importe de comprendre c'est que le manifeste polarise, il est là pour diviser. Le manifeste au cinéma a toujours été à l'encontre du système et des normes. L'objectif latent du manifeste est donc de faire sortir le cinéma de son état actuel pour le faire avancer (ou revenir en arrière) à travers une prescription. II s'agit d'une prise de parti dans un conflit artistique, politique, esthétique. Le manifeste n'existe pas comme la proposition spontanée 
d'un programme mais, bien au contraire, il émerge comme une réponse souvent violente et fermée vis-à-vis d'un état de choses. Il a donc des ennemis avant même d'avoir des amis.

Notre intention est de mettre en parallèle deux mouvements qui ont émergé respectivement au milieu et à la fin du $20^{\mathrm{e}}$ siècle: la Nouvelle Vague qui, entre les années 1950 et 1960, a concentré l'intérêt du monde du cinéma en se révoltant contre la tradition et Dogme 95, qui en a suivi l'exemple. Le premier est un mouvement de libération, d'ouverture, né du besoin de renouveler une pratique artistique figée, presque automatique et trop dépendante du marché; le deuxième est prêt à faire des sacrifices et à s'imposer un régime strict afin de retrouver une sincérité perdue, loin des artifices et du spectaculaire. La Nouvelle Vague est donc un groupe jamais complètement et définitivement constitué, dont l'ambiguïté des participants par rapport à l'existence et à la cohérence du collectif n'a jamais cessé, mais qui a de façon indéniable revitalisé le cinéma. Quant à Dogme 95, il est revenu en arrière pour se servir d'un outil en désuétude, le manifeste.

\section{Histoire d'un manifeste négatif. Le cri contre la «Tradition de la Qualité »}

Au début de la période connue comme "l'âge d'or du cinéma français », entre 1947 et 1957, au cours de laquelle une moyenne de 109 films par an est produite/1, Le Silence de la mer de Jean-Pierre Melville représente un antécédent direct de la Nouvelle Vague. Le réalisateur sort dans la rue avec une caméra, tournant dans un décor naturel, sans l'autorisation de Vercors (auteur de la nouvelle éponyme qui inspire le film), sans vedettes, avec une équipe de collaborateurs sans carte professionnelle et avec un budget réduit/2. Parallèlement, la publication de l'article d'Alexandre Astruc « Naissance d'une nouvelle avant-garde/3 ", peut être qualifiée de prémanifeste de la Nouvelle Vague. Astruc prophétise un cinéma qui se constitue comme un moyen d'expression nouveau et indépendant. Au-delà de l'anecdote et du contenu thématique des films, il s'intéresse au rapport du cinéma avec le théâtre, la littérature ou la peinture et accorde une place centrale à la figure du réalisateur, de l'auteur, qui « écrit avec sa caméra, comme un écrivain écrit avec un stylo/4 ». Pour Astruc, le cinéma est une façon d'exprimer la pensée avant d'être un procédé pour raconter des histoires; il met en valeur la notion d'auteur face à une tyrannie du public et du producteur, qui préfère un spectacle séduisant à la promotion d'une vision plus particulière et personnelle.
/1 René Bonnell, Le Cinéma exploité, Paris, Seuil, 1978, p. 165.

/2 Le budget dont dispose Melville correspond alors à un sixième du budget habituel pour un film, à savoir 9 millions au lieu de 55 millions d'anciens francs. Michel Marie, La Nouvelle Vague et son film manifeste À bout de souffle, Paris, Armand Colin, 2009, p. 47.

/3 Alexandre Astruc, "Naissance d'une nouvelle avant-garde: la caméra-stylo » (1948), dans Du stylo à la caméra... et de la caméra au stylo: Écrits 1942 1984, Paris, L’Archipel, 1992.

14 ibid., p. 327. 
/5 François Truffaut, "Une certaine tendance du cinéma français ", Cahiers du cinéma, $\mathrm{n}^{\circ} 31$, janvier 1954, p. 15-29.

/6 « Ces dix ou douze films constituent ce qu'on a joliment appelé la Tradition de la Qualité, ils forcent par leur ambition l'admiration de la presse étrangère, défendent deux fois l'an les couleurs de la France à Cannes et à Venise où, depuis 1946, ils raflent assez régulièrement médailles, lions d'or et grands prix. ». ibid., p. 15 .

\section{/7 ibid., p. 23.}

/8 François Truffaut, "Vous êtes tous témoins dans ce procès: le cinéma français crève sous les fausses légendes ", Arts n 619, 15 mai 1957, p. 1.

/9 François Truffaut, "Une certaine tendance du cinéma français ", op. cit., p. 26.
Quand François Truffaut publie «Une certaine tendance du cinéma français/5 ", en janvier 1954, il rencontre des résistances de la part d’André Bazin et de Jacques Doniol-Valcroze, les rédacteurs en chef des Cahiers du Cinéma. La raison en est l'attaque directe visant Claude Autant-Lara, Jean Delannoy, Yves Allegret, René Clément et les scénaristes Jean Aurenche et Pierre Bost, représentant tous la «Tradition de la Qualité/6». La diatribe de Truffaut a pour objectif de démonter la dite "Tradition » qui, derrière une supposée fidélité à l'esprit des œuvres littéraires adaptées au cinéma et le talent des scénaristes pour y parvenir, cache plutôt une manière uniforme et automatique de faire du cinéma, un prétendu réalisme qui n'est qu'une vulgaire caricature de la réalité: "Il n’y a guère que sept ou huit scénaristes à travailler régulièrement pour le cinéma français. Chacun de ces scénaristes n'a qu'une histoire à raconter et comme chacun n'aspire qu'au succès des “deux grands”, il n'est pas exagéré de dire que les cent et quelques films français réalisés chaque année racontent la même histoire: il s'agit toujours d'une victime, en général un cocu. (Ce cocu serait le seul personnage sympathique du film s’il n'était toujours infiniment grotesque: Blier-Vilbert, etc.). La rouerie de ses proches et la haine que se vouent entre eux les membres de sa famille, amène le "héros" à sa perte; l'injustice de la vie, et, en couleur locale, la méchanceté du monde (les curés, les concierges, les voisins, les passants, les riches, les pauvres, les soldats, etc.)/7 ». C'est dans cette critique ironique et colérique que le manifeste prend forme, dans un attaque à l'encontre d'un cinéma sclérosé, répétitif et en crise de courage et de virilité (comme Truffaut dira lui-même trois ans plus tard/8). Mais en même temps, son article de 1954 contient, résume et exprime les principes fondateurs de celle qui deviendra une esthétique clé pour la Nouvelle Vague. Bien que l'accent de sa critique vise ce qui ne va pas dans le cinéma contemporain, il exalte le travail d'un groupe de réalisateurs (Jean Renoir, Max Ophuls, Robert Bresson, Jacques Becker, Jacques Tati, Jean Cocteau) sous l'expression de "cinéma d'auteur/9 ». C'est de cette façon que le manifeste divise. Truffaut établit clairement deux groupes opposés: ceux qui font des films d'une manière plutôt industrielle et machinale et les «vrais » réalisateurs-auteurs qui souhaitent exprimer leur vision particulière à travers le seul outil qui leur est propre: le cinéma. «Je ne conçois d'adaptation valable qu'écrite par un homme de cinéma. Aurenche et Bost sont essentiellement des littérateurs et je leur reprocherai ici de mépriser le cinéma en le sous-estimant. Ils se comportent vis-à-vis du scénario comme l'on croit rééduquer un délinquant en lui trouvant du travail, ils croient toujours avoir "fait le maximum" pour lui en le parant des subtilités, de cette science des 
nuances qui font le mince mérite des romans modernes. Ce n'est d'ailleurs pas le moindre travers des exégètes de notre art que de croire l'honorer en usant du jargon littéraire/10. ».

Truffaut appelle à la reconnaissance du cinéma comme un art indépendant, à l'authenticité de ses créateurs et au respect d'une spécificité artistique. Sa dénonciation est aussi une revendication du travail de réalisateur. En proposant la constitution de deux groupes différenciés et fortement opposés, il cherche à soutenir la production des auteurs en les rassemblant sans pour autant leur faire perdre leur singularité, c'est-à-dire leur indépendance et originalité. Le texte de Truffaut, s'il n'a pas été ouvertement construit comme un manifeste, le devient grâce à son affirmation comme point de départ d'une nouvelle époque cinématographique, incapable de partager son espace vital avec l'ancienne; émergeant au sein d'une industrie majoritaire et dominante et contre celle-ci. L'intransigeance de son propos et la radicalité de sa démarche ne se limitent pas à opposer deux façons de faire du cinéma, mais vise aussi à anéantir l'ancienne pour permettre à la nouvelle de se développer. En créant ces deux extrêmes, il invite les réalisateurs contemporains et futurs à choisir entre soutenir et poursuivre la tradition ou intégrer la Nouvelle Vague - qui portera ce nom à partir de 1958/11. Mais, comment participer concrètement au renouveau du cinéma?

Même si «Une certaine tendance du cinéma français » donne des idées précises sur le rôle du réalisateur, le texte est loin d'offrir une prescription sur des aspects techniques. La négativité du manifeste de Truffaut tient au fait de dire ce qu'il ne faut pas faire, plutôt que ce qu'il faut faire du point de vue des méthodes et des procédés. A posteriori, à partir de certaines techniques utilisées par Melville dans Le Silence de la mer, d'autres principes décrits par Truffaut et des méthodes et pratiques vues dans les premiers films du mouvement, nous pouvons établir quelques principes connus comme la base esthétique de la Nouvelle Vague:

«1. L'auteur-réalisateur est également le scénariste du film: la création cinématographique n'est pas un processus collectif mais la matérialisation du regard de l'auteur, de sa mise en scène;

2. Ne pas utiliser un découpage technique strict, mais laisser une marge importante à l'improvisation dans la conception des séquences, du cadrage, des dialogues, et du jeu des acteurs;

3. Se servir de décors naturels;

4. Travailler avec une équipe réduite au minimum;

5. Adopter le son direct plutôt que la postsynchronisation;

6. Ne pas utiliser des éclairages additionnels et en général un matériel trop lourd. Opter pour le léger et rapide;
/10 ibid., p. 20.

/11 « Le terme "Nouvelle Vague" paraît pour la première fois dans L'Express du 3 octobre 1957. Il sert de titre au Rapport sur la jeunesse que commente Françoise Giroud, une journaliste de cet hebdomadaire. [...] Pierre Billard, en février 1958 [ramasse ['expression et] dans Cinéma 58, l'applique pour la première fois au désir de renouvellement que manifestent les jeunes du cinéma français. C'est au Festival de Cannes, en 1959, que le terme se généralise pour qualifier les nombreux jeunes réalisateurs présents. ". Jean Douchet, Nouvelle Vague, Paris, Hazan, 1998, p. 164. 
/12 Michel Marie, La Nouvelle Vague et son film manifeste À bout de souffle, Paris, Armand Colin, 2009, p. 66.

/13 Le Coup du Berger (1956), court-métrage de Jacques Rivette, produit par Claude Chabrol; Le Beau Serge (1958), de Claude Chabrol, autoproduit; Les Coussins (1959) de Claude Chabrol; Les 400 coups (1959) de François Truffaut; À Bout de souffle (1959) de Jean-Luc Godard; L'eau à la bouche (1959), signé Jacques DoniolValcroze; Le Signe du lion (1959) d'Éric Rohmer; Le Bel Âge (1960), deuxième film de Pierre Kast; Le Petit soldat (1960) de Jean-Luc Godard; Tirez sur le pianiste (1960), deuxième film de François Truffaut; Paris nous appartient (1961), premier long métrage de Jacques Rivette.
7. Filmer des non-professionnels ou des nouveaux acteurs. Cela pour des raisons économiques mais aussi parce que le réalisateur peut diriger de manière plus libre/12. »

Ainsi, même lorsque des aspects techniques sont mélangés à des questions théoriques, il s'agit de choix qui cherchent à réduire la brèche entre le cinéma reconnu comme professionnel et un «amateurisme » moins réglementé qui essaie de s'évader des conditions imposées par le modèle industriel et commercial du cinéma. Cependant, comme pour tout manifeste, les postulats sont susceptibles d'être interprétés, enrichis, complétés. Ainsi, la quasi-totalité des réalisateurs qui font partie de la Nouvelle Vague ne s'engagent que partiellement dans ces principes. Dans le cas de Truffaut lui-même, Chabrol, Varda, Demy ou Kast, l'appartenance au mouvement répond plutôt à un accord intellectuel et à d'autres caractéristiques comme le petit budget, le caractère autobiographique de ces films, la liberté narrative, etc., et pas forcément aux techniques cinématographiques (en particulier celles du montage et son direct), à l'absence de découpage et au fait d'être son propre scénariste, déjà cités. Tous ces réalisateurs sont d'accord avec les nouvelles méthodes, mais ils ne les pratiquent pas nécessairement, préférant tourner à partir d'un scénario préalable et faisant usage de la postsynchronisation, par exemple.

Les premiers films aujourd'hui reconnus comme faisant partie du mouvement/13 ont sans doute en commun le travail critique des réalisateurs au sein des Cahiers du Cinéma et le fait qu'il s'agit de leur premier ou deuxième film. Mais malgré quelques aspects qui les rapprochent, ils sont loin d'une uniformité esthétique. Parmi eux, À Bout de souffle de Jean-Luc Godard, réalisé à partir d'une idée de Truffaut, incarne les nouvelles idées du mouvement et devient la référence incontestable, un deuxième manifeste.

\section{Le deuxième manifeste: $\grave{A}$ bout de souffle}

Par rapport aux premiers films de Chabrol et Truffaut, Jean-Luc Godard opère une incontestable révolution technique avec À bout de souffle (1959). Véritable manifeste cinématographique de la Nouvelle Vague, ce film en contient les caractéristiques et audaces techniques. Un montage basé sur la discontinuité, les plans sur plan, le temps des plans-séquences, le langage cru et plus direct, les bruits de la ville, la musique, la fragmentation, la vitesse de tournage, le budget réduit, la spontanéité, les discours face à la caméra, la narration impersonnelle, le rapport aux personnages, l'apparition de la subjectivité du réalisateur, etc., sont autant de moyens concrets 
pour le film de lutter contre la sclérose esthétique et économique du cinéma de la tradition. Ces techniques constituent une nouvelle manière de réaliser et de voir le cinéma. Les faux raccords et les sauts permettent au film de se raccourcir en gardant le nombre de séquences, tout en accélérant le rythme. Ce dont il est question c'est l'expression personnelle du réalisateur et la réinterprétation du rapport classique entre les personnages, les situations et la longueur des plans. Godard fait un montage accéléré qui devient une référence de la Nouvelle Vague et s'oppose au montage parallèle ou contrapuntique plus traditionnel. Si le film donne l'impression de "sauter ", ce qui peut être dû à des raisons économiques plus qu'esthétiques, cela répond aussi à la conception particulière que Godard a du cinéma.

Le film est un deuxième manifeste qui complète le premier. Dans l'article de Truffaut de 1954, il y a la déclaration de guerre mais il manque la proposition d'un plan à suivre pour changer l'état de l'art cinématographique. Godard donne en revanche un exemple de ce que peut donner le cinéma d'auteur après Renoir ou Bresson. Le film, qui incorpore les inquiétudes de l'époque présentées par Truffaut, est le résultat du travail d'un «non professionnel » qui aboutit à la mise en scène par le biais de la critique, de la connaissance théorique du cinéma. Godard fait la transition de la critique à la réalisation comme s'il s'agissait de la même chose, le mouvement est continuel, sans rupture : le théoricien devient auteur. Les années passées au Cahiers du Cinéma ont renforcé l'idée de Godard selon laquelle le film est l'œuvre d'un seul homme. Même s'il reprend un scénario de Truffaut et que Claude Chabrol est son conseiller technique, $\grave{A}$ bout de souffle incarne sa perception du métier de réalisateur et, en même temps, montre un monde qu'il connaît bien. C'est dans cette mesure que nous pouvons parler d'un film personnel, où la subjectivité du réalisateur est mise en avant. "Dans À bout de souffle dont le sujet est dérivé de la mythologie américaine - les clins d'œil à Humphrey Bogart, à Jean Pierre Melville, aux westerns ou à William Faulkner se rapportent aux partis-pris de Jean-Luc Godard et non à ceux de Michel Poiccard/14 ».

L'écriture du film, la conception de sa mise en scène, comprennent et exhibent les principes de la Nouvelle Vague. La transgression du champ-contrechamp et les mouvements de la caméra sèment les bases d'une grammaire filmique qui permet d'éclater le code établi. Non seulement ce film correspond à l'esthétique de la Nouvelle Vague, mais les films suivants sont fortement influencés par $A$ bout de souffle, même s'ils n'essaient pas forcément de l'imiter. Le film constitue ainsi un manifeste et est iconique parce qu'il comporte les
/14 Claire Clouzot, Le Cinéma français depuis la Nouvelle Vague, Paris, Nathan, 1972, p. 30. 
/15 François Truffaut, France-Observateur $\mathrm{n}^{\circ}$ 501, 3 décembre, 1959.

/16 Michel Marie, La Nouvelle Vague et son film manifeste À bout de souffle, op. cit., p. 16.

/17 ibid., p. 18. méthodes et anti-méthodes qui donnent cohérence au mouvement, qui deviennent la référence et qui permettent de parler d'un avant et d'un après la Nouvelle Vague. Au-delà de l'ordre chronologique, c'est le film fondateur du courant, il brise et triture les composants du cinéma pour le réinventer, pour déclarer en même temps sa mort et sa renaissance.

Le succès économique, médiatique et auto-promotionnel d'À bout de souffle est accompagné par son importance technique et esthétique et donne à l'étiquette de « Nouvelle Vague » un point de repère. C'est le travail d'un aventurier, celui que Truffaut avait prédit deux ans auparavant. La provocation, la fraîcheur et l'innovation dans ses procédés marquent la distance par rapport à la génération précédente. Dès qu'ils possèdent un nom, une doctrine générale (la politique des auteurs), un programme esthétique consistant à faire des films personnels, des théoriciens comme André Bazin, Astruc et Truffaut, les Cahiers du Cinéma comme support éditorial, des auteurs, des films, des amis et des ennemis, les deux manifestes, celui publié par Truffaut et celui filmé par Godard, complètent leur fonction. Maintenant, c'est aux réalisateurs de déclarer leur adhésion ou non. Dès 1959, Truffaut remarque: "ce slogan qui, à mon avis, ne correspondait pas à une réalité, dans la mesure où, à l'étranger, on a pu croire par exemple qu'il y avait une association de jeunes cinéastes français qui se réunissaient à des dates régulières et qui avaient un plan, une esthétique commune, alors qu'il n'en était rien et qu'il ne s'agissait que d'un rassemblement fictif, tout à fait extérieur/15 ». Godard aussi parle d'un « amalgame abusif et très superficiel/16 ». Cependant, après avoir été attaqués par les réalisateurs plus classiques, Truffaut et Godard affirmeront leur appartenance au mouvement et l'originalité de son esthétique.

Après les premiers succès, les déconvenues arrivent avec la censure du Petit soldat de Godard, l'échec d'Une femme est une femme, des Carabiniers (qui compte seulement 2800 spectateurs en deux semaines d'exploitation), des Bonnes femmes de Chabrol et de Tirez sur le pianiste de Truffaut. Ce dernier attend trois ans pour réussir Fahrenheit 451, tout comme Jacques Rivette pour distribuer Paris nous appartient et Eric Rohmer pour réaliser Le signe du lion. Il est difficile de dater la Nouvelle Vague avec précision. Nous pouvons dire cependant qu'elle a commencé soit en 1956 avec le court métrage Le Coup du Berger de Jacques Rivette, soit en 1958 avec le premier long métrage de Claude Chabrol, et qu'elle a duré jusqu'en 1963/17. Cette période est suffisante pour consolider l'une des plus importantes «écoles» dans l'histoire du cinéma. Elle élabore une stratégie d'autopromotion réussie et compte sur une figure qui « pratique 
une rupture totale/18 $"$, qui incarne les innovations techniques et conceptuelles: Godard.

De nombreux réalisateurs et mouvements de différents pays peuvent se dire héritiers de la Nouvelle Vague/19. Un cas significatif a lieu quarante-deux ans après la fin de la Nouvelle Vague, à Copenhague.

\section{Dogme 95}

«En 1960, c'en était trop! Le cinéma était mort et appelait à une résurrection. Le but était juste mais pas les moyens. La Nouvelle Vague ne fut qu'un clapotis qui s'échoua sur le rivage pour se transformer en boue.

Les slogans d'individualisme et de liberté ont momentanément créé des œuvres, mais n’ont apporté aucun changement. La vague était à vendre, les réalisateurs aussi. La vague ne fut pas plus forte que ceux qui l'avaient créée. Le cinéma anti-bourgeois devint bourgeois, parce qu'il était basé sur des théories reposant sur une conception bourgeoise de l'art. Le concept d'auteur issu du romantisme bourgeois était donc... faux! Pour Dogme 95, le cinéma n'est pas individualiste! [...] Pour Dogme 95 le film n'est pas illusion/20! ».

Cet extrait du manifeste de Dogme 95, écrit au Danemark mais lu au Théâtre de l'Odéon à Paris le 20 mars 1995, fait partie de la proclamation officielle du mouvement conçu par les réalisateurs danois Thomas Vinterberg et Lars von Trier. Ce coup de marketing bien planifié, pensé pour la célébration du centenaire du cinéma, inclut donc une attaque contre le cinéma mondial des quarante dernières années, une lecture du « vœu de chasteté » et même le départ inattendu et précipité de Lars von Trier après la lecture, comme pour laisser planer le mystère. Dans ce cas, l'ennemi n'est pas un cinéma national devenu industriel, commercial et qui perd son caractère artistique, mais les superproductions anglo-saxonnes qui envahissent les salles et, par un usage exagéré des effets spéciaux, dénaturent la capacité expressive du cinéma. La solution mise en avant par ses créateurs est d'accepter certaines règles, selon un décalogue sous la forme d'un vœu de chasteté:

« Je jure de me soumettre aux règles qui suivent telles qu'édictées et approuvées par Dogme 95.

1. Le tournage doit être fait sur place. Les accessoires et décors ne doivent pas être amenés (si on a besoin d'un accessoire particulier pour l'histoire, choisir un endroit où cet accessoire est présent).

2. Le son ne doit jamais être réalisé à part des images, et inversement (aucune musique ne doit être utilisée à moins qu'elle ne soit jouée pendant que la scène est filmée).
/18 Glauber Rocha, interview par Marcel Martin, Cinéma 71 n 160 , novembre 1971.

/19 Nous pouvons mentionner les italiens Pier Paolo Pasolini, Bernardo Bertolucci, Nanni Moretti, le brésilien Glauber Rocha, le polonais Jerzy Skolimowski, l'allemand Wim Wenders, l'iranien Abbas Kiarostami, les taïwanais Hou Hsiao Hsien et Edward Yang, le chinois John Woo et les français Jean Eustache, Leos Carax ou Philippe Garrel, parmi beaucoup d'autres.

/20 Manifeste Dogme 95, trad. C. Chatelet, dans « Dogme 95 : un mouvement ambigu, entre idéalisme et pragmatisme, ironie et sérieux, engagement et opportunisme », 1895. Mille huit cent quatrevingt-quinze, $\mathrm{n}^{\circ} 482006$, [http://1895.revues. org/341], consulté le 24 avril 2015. 
/ 21 Vœu de chasteté, ibid.

/22 Il y a donc deux documents. Le premier est le manifeste et le deuxième son programme: le vœu de chasteté.
3. La caméra doit être portée à la main. Tout mouvement, ou nonmouvement possible avec la main est autorisé. (Le film ne doit pas se dérouler là où la caméra se trouve; le tournage doit se faire là où le film se déroule).

4. Le film doit être en couleurs. Un éclairage spécial n'est pas acceptable. (S'il n'y a pas assez de lumière, la scène doit être coupée, ou une simple lampe attachée à la caméra).

5. Tout traitement optique ou filtre est interdit.

6. Le film ne doit pas contenir d'action de façon superficielle. (Les meurtres, les armes, etc. ne doivent pas apparaître).

7. Les détournements temporels et géographiques sont interdits. (C'est-à-dire que le film se déroule ici et maintenant).

8. Les films de genre ne sont pas acceptables.

9. Le format de la pellicule doit être le format académique $35 \mathrm{~mm}$. 10. Le réalisateur ne doit pas être crédité /21. ».

Il s'agit maintenant d'un véritable manifeste, d'un texte planifié avec la claire intention d'être suivi, de devenir un guide et une référence unique et incontestable politiquement et esthétiquement. Le texte est aussi signé par une collectivité même si elle n'est composée que de deux personnes. Le choix de s'exprimer à travers un manifeste et l'usage d'une rhétorique particulière renvoient anachroniquement à une époque passée. À travers un outil qui n'est plus d'actualité, Lars von Trier et Thomas Vinterberg adoptent la forme discursive du manifeste comme un défi. De cette façon et avec une dose d'ironie, ils intègrent l'histoire des mouvements et des revendications artistiques de manière polémique.

Le mouvement, contrairement à la Nouvelle Vague, prend forme à partir d'un événement spécifique. Tout est daté, signé, organisé (énuméré) et les principes sont fermes. Toutefois, l'idée de faire des films avec une camisole de force n'est pas seulement une façon de revitaliser l'expressivité de l'art cinématographique, mais aussi de construire une identité au sein d'un groupe; de résister à l'invasion tout en profitant d'un choc médiatique. L'expérience proposée aux réalisateurs est de faire une espèce de pause dans les procédés réguliers du cinéma pour retrouver sa créativité loin de la machinerie industrielle. Le langage religieux, voire militaire, très rigide, très strict, fait penser à une secte plus qu'à un groupe d'artistes. S'éloigner des normes édictées est un pêché, une faute face aux frères qui ont aussi fait ce vœu de chasteté, qui ont accepté le dogme. Le refus de soi, la négation de l'individualité pour accueillir une mission plus importante, plus vaste : la discipline, l'abstinence, la recherche symbolique de la pureté, de la vérité, sont les stratégies de ce nouveau double manifeste/22. Le cinéaste doit abandonner ses habitudes, se 
nettoyer, se purifier pour retrouver l'essence de son métier, qui n'est pas de tromper le public mais de lui montrer un chemin possible, loin de la subjectivité artistique bourgeoise mais proche de la vérité la plus simple, sans artifices.

En épurant le film des astuces cinématographiques, en rapprochant la scène filmée de la scène qui va apparaître finalement, Dogme 95 rêve d'un retour aux sources. Tout comme la Nouvelle Vague, Dogme 95 veut se libérer des artifices psychologiques et techniques pour retrouver la justesse et la légitimité. Le manifeste danois est toujours contradictoire: il critique un modèle en lui en opposant un autre, il promet la liberté à travers l'obéissance et l'acceptation des règles, il cherche l'innovation en niant les outils nouveaux et aspire à la vérité des choses, au-delà des mensonges et subterfuges. Dogme 95 réfute l'œuvre, l'art et l'individu. Désormais, le travail est celui d'une équipe; le réalisateur doit s'effacer et ne pas produire de « l'art » mais opérer une sorte de retour aux sources, à l'authenticité. Tous ces éléments permettent de penser que le manifeste et le vœu de chasteté sont construits avec une importante dose d'ironie, ainsi que le met en valeur Claire Chatelet: «Le terme de “jeu” ne serait-il pas finalement le plus approprié pour résumer Dogme 95? Au sens d'activité ludique d'abord: l'ambition “purificatrice” et la stratégie "minimaliste” du mouvement manifestent clairement son désir ludique de renouveler le cinéma, de réinventer la pratique cinématographique en suivant un certain nombre de contraintes qui sont en réalité autant de règles du jeu, un jeu qui consiste précisément à se conformer à un ensemble de règles pour se libérer d'autres règles - celles du cinéma conventionnel “dominant”. Au sens de comédie ensuite (jouer un rôle): en mêlant des idées religieuses aux stratégies et aux formes de l'avant-garde artistique (constitution d'un groupe, positionnement en termes d'opposition, élaboration d'une doctrine sous la forme d'un manifeste provocateur, proposition d'un programme d'action à visée internationale, mise en cause du statut d'œuvre et de l'idéologie bourgeoise de l'art, utilisation concertée des médias pour une visibilité maximale), Dogme 95 s'est construit une identité singulière et nécessairement polémique, et s'est garanti lui-même les moyens de sa reconnaissance/23 ".

Très tôt dénoncé par certains comme une arnaque publicitaire et étiqueté par d'autres comme du génie et surtout comme l'opportunité de produire et d'être distribué grâce au label Dogme, le manifeste produit ses deux premiers films trois ans plus tard: Festen de Vinterberg et Idioterne (Les Idiots) de Lars von Trier; le premier gagne le Grand Prix du Jury de Cannes mais le deuxième génère une forte polémique. Dogme étant un mouvement de portée internationale, il
/23 Claire Chatelet, «Dogme 95: un mouvement ambigu... », op. cit. 
y eut très rapidement des films en France (Lovers de Jean-Marc Barr), aux États-Unis (Julien Donkey-Boy de Harmony Korine), en Corée du Sud (Interview de Daniel Byun), en Argentine (Fuckland de José Luis Marques), mais aussi en Italie, en Suisse, en Norvège, en Belgique, en Espagne, au Chili. Pour de nombreux réalisateurs, Dogme 95 est l'occasion d'une première expérience cinématographique, qui s'est avérée aussi être leur dernière. Certaines œuvres ont été récompensées par des prix ou des reconnaissances internationales mais à l'exception de Festen, Les Idiots, Mifune, Julien Donkey-Boy, Strass et d'une partie des films danois, aucun d'entre eux n'a eu un impact significatif dans la distribution et l'exploitation en salles, ni auprès de la critique.

Cependant, la médiatisation est réussie et, en plus d'accorder un certificat Dogme 95 numéroté à chaque film qui promet de s'adapter aux règles du jeu et dont les auteurs acceptent de verser 10000 couronnes danoises (aujourd'hui près de 1300 euros), un site Internet officiel a été créé afin de faciliter la systématisation des démarches. Les lois du vœu de chasteté restent fixes mais leur interprétation est ouverte. Cela aboutit à un mouvement flexible et varié qui compte des films politiques, des documentaires, ainsi que d'autres plus intéressés par l'exploration artistique, esthétique ou bien sociale. Dans tous les cas, la rénovation formelle du cinéma passe par un travail non individuel mais collectif et libre, toujours en constante création, dont l'improvisation et la spontanéité sont les axes majeurs.

En profitant de la démocratisation du cinéma, conséquence de la "tempête technologique » dans laquelle nous vivons, Dogme 95 croit qu'il ne suffit pas que tout le monde puisse faire des films, mais qu'il faut porter un même nom et uniforme, pour se rebeller contre l'individualisme décadent. Un travail en groupe dont l'identité est commune et non une affaire d'ego, dont le réalisateur est un «frère » ou une " sœur » qui travaille en équipe pour communiquer de façon plus sincère et directe, sans se concentrer sur les actions superficielles, sans prendre en compte son goût personnel, ni les mœurs esthétiques ni sa condition d'artiste, sans avoir pour objectif la création d'une œuvre. Dogme 95 refuse de prolonger l'idée de l'artiste comme auteur, promulguée par la Nouvelle Vague. Pour les réalisateurs danois, l'art est bourgeois. Le cinéma doit se vider des contraintes esthétiques artificielles, de la soif de rendre tout extraordinaire, de l'utilisation inutile de gags techniques, des clins d'œil artistiques.

Cependant, si l'idée d'origine des fondateurs est d'inspirer un mouvement permanent de lutte et de rébellion, la société spectaculaire a rapidement récupéré le phénomène pour en faire une convention, 
jusqu'à réduire le programme de Dogme 95 à l'emploi de la caméra à l'épaule. Ni les fondateurs ni les promoteurs et participants du Dogme 95 n'ont jamais appliqué l'intégralité des dix «commandements » présents dans le vœu de chasteté, mais ils ont essayé de s'en approcher le plus possible. Dix ans après la soirée au Théâtre de l'Odéon, le 20 mars 2005 , le couple fondateur a annoncé qu'ils ne prenaient plus la responsabilité du groupe, mais encourageaient les réalisateurs à poursuivre leurs travaux et à accepter les règles s'ils le veulent.

\section{En guise de conclusion}

Nous avons d'un côté deux manifestes "involontaires », un article et un film qui, entre 1954 et 1959, donnent naissance, cohérence et solidité à un groupe de réalisateurs. Ceux-ci, malgré leurs récurrentes contestations et hésitations initiales, ont, dans la plupart des cas, fini par admettre la validité du mouvement, sinon comme programme esthétique, au moins comme volonté de produire en dehors de l'industrie. La Nouvelle Vague est un courant artistique attaché à des conceptions critiques, politiques et parfois esthétiques et techniques, communes d'abord à un groupe de critiques et de cinéphiles, puis de réalisateurs les plus influents de leur génération. Les manifestes de la Nouvelle Vague ont eu la seule forme qu'ils pouvaient avoir: une forme en mouvement, non figée, plus libre, claire dans ses propos mais ouverte à l'interprétation. De l'autre côté, Dogme 95 qui, de manière très significative, commence par citer la Nouvelle Vague comme antécédent immédiat d'un essai de rénovation du cinéma, construit également deux manifestes, mais de façon plus stricte, presque militaire, bien qu'ironique en même temps. Si pour la Nouvelle Vague, et pour À bout de souffle en particulier, tout était permis, pour Dogme 95 beaucoup de choses sont interdites; mais il s'agit de deux manières différentes d'aborder la réalisation face à l'état du cinéma.

Les deux mouvements répondent donc à l'apparition de conditions qui ont permis aux jeunes réalisateurs de tourner leurs premiers films. Les uns aident les autres. Le premier succès de Chabrol a encouragé non seulement d'autres réalisateurs mais aussi quelques producteurs à se diriger vers d'autres possibilités; le premier succès de Truffaut puis le deuxième de Chabrol offrent à Godard la possibilité de tourner À Bout de souffle; au Danemark, les aides financières accordées à Dogme 95 ont motivé et donné une nouvelle impulsion à la production nationale. Les manifestes "involontaires» de la Nouvelle Vague s'opposent au lancement stratégique du Dogme 95. 
Mais dans les deux cas, la notion de manifeste va permettre l'existence et surtout le développement des groupes, au sein desquels les réalisateurs trouvent un appui économique, esthétique et conceptuel. Pour la plupart d'entre eux, c'est la seule façon de produire des films.

La Nouvelle Vague et Dogme 95 ont opéré un renouvellement de la création cinématographique. Même si beaucoup de films ne sont pas bien distribués, si leur qualité n'est pas optimale ou si pour leurs réalisateurs il n'y a pas eu de deuxième film, l'histoire du cinéma se compose tout autant de ses tentatives que de ses échecs. Plusieurs décennies plus tard et au-delà des héritiers directs, il est évident que l'ampleur et l'influence de ces mouvements échappe à la classification et que la recherche de « l'esprit de pauvreté » mentionné par Rivette n'était pas seulement une question d'argent. Le cinéma qui résiste au commerce est aussi plus libre, car pour les deux mouvements même si on n'a pas la formation nécessaire, si on n'a pas les ressources économiques - et surtout au-delà des tendances esthétiques et artistiques de l'époque - il faut continuer à faire des films.

\section{Carlos Tello}

